\title{
Recent Progress on Phase Equilibrium Calculation in Subsurface Reservoirs using Diffuse Interface Models
}

\author{
Tao Zhang ${ }^{1,2}$, Yiteng $\mathrm{Li}^{2}$, Jianchao $\mathrm{Cai}^{1}$, and Shuyu $\mathrm{Sun}^{2,1, *}$ \\ 1 Institute of Geophysics and Geomatics, \\ China University of Geosciences, Wuhan 430074, P.R. China \\ tao.zhang.1@kaust.edu.sa \\ caijc@cug. edu.cn \\ shuyu. sun@kaust.edu.sa \\ 2 Physical Science and Engineering Division, \\ King Abdullah University of Science and Technology, \\ Thuwal 23955-6900, Saudi Arabia \\ tao.zhang.1@kaust.edu.sa \\ yiteng.li@kaust.edu.sa \\ shuyu. sun@kaust.edu.sa
}

\begin{abstract}
Compositional multiphase flow in subsurface porous media is becoming increasingly attractive due to issues related with enhanced oil recovery, greenhouse effect and global warming, and the urgent need for development in unconventional oil/gas reservoirs. One key effort prior to construct the mathematical model governing the compositional multiphase flow is to determine the phase compositions of the fluid mixture, and then calculate other related physical properties. In this paper, recent progress on phase equilibrium calculations in subsurface reservoirs have been reviewed and concluded with authors' own analysis. Phase equilibrium calculation is the main approach to perform such calculation, which could be conducted using two different types of flash calculation algorithms: the NPT flash and NVT flash. NPT flash calculations are proposed early, well developed within the last few decades and now become the most commonly used method. However, it fails to remain the physical meanings in the solution as a cubic equation, derived from equation of state, is often needed to solve. Alternatively, NVT flash can handle the phase equilibrium calculations as well, without the pressure known a priori. Recently, Diffuse Interface Models, which were proved to keep a high consistency with thermodynamic laws, have been introduced in the phase calculation, incorporating the realistic equation of state (EOS), e.g. Peng-Robinson EOS. In NVT flash, Helmholtz free energy is minimized instead of Gibbs free energy used in NPT flash, and this energy density is treated with convex-concave splitting technique. A semi-implicit numerical scheme is designed to process the dynamic model, which ensures the thermodynamic stability and then preserve the fast convergence property. A positive definite coefficient matrix is
\end{abstract}

${ }^{*}$ Corresponding author: shuyu.sun@kaust.edu.sa 
designed to meet the Onsager Reciprocal Principle so as to keep the entropy increasing property in the presene of capillary pressure, which is required by the thermodynamic laws. The robustness of the proposed algorithm is verified via two numerical examples, one of which has up to seven components. In the complex fluid mixture, special phenomena could be capture from the global minimum of TPD functions as well as the phase envelope resulted from the phase equilibrium calculations. It can be found that the boundary between the single-phase and vaporliquid phase regions will move in the presence of capillary pressure, and then the area of each region will change accordingly. Some remarks have been concluded at the end, as well as suggestions on potential topics for future studies.

Keywords: multiphase multicomponent flow, NVT flash, capillarity, phase equilibrium, diffuse interface models

\section{Introduction}

Modeling and simulation of the subsurface multiphase fluid flow in porous media have a variety of applications, including reservoir engineering and environmental protection. In petroleum industry, it can be used to simulate the various exploitation stages of an oilfield and optimize the development plan for oil recovery maximization $[2,5,16,17,24,22,25]$. On the other hand, for the increasingly concerned environmental issues (e.g. greenhouse gas emission and pollutant disposal), the simulation of subsurface flow could shed light on the trapping mechanisms of $\mathrm{CO}_{2}$ sequestration $[7,8]$ or the migration behaviors of underground pollutants (e.g. nuclear waste), which helps to either remarkably reduce the $\mathrm{CO}_{2}$ emission or controllably dispose the environmentally hazardous wastes. In addition, the production of unconventional oil and gas resources has experienced a significant growth in all over the world and successfully becomes an important energy supply, due to the rapid production decline of conventional reservoirs [15]. In order to maximize the oil prodcution from conventional and unconventional reservoir or resolve the aforementioned environment issues, it is great demand of the accurate numerical model of subsurface multiphase fluid systems, as well as the robust and efficient computational algorithm.

The subsurface flow model is often formulated based on varieties of conservation laws, including mass conservation, momentum conservation and energy conservation. One key effort to grab these conservation properties is to determine the phase composition, that is, to find out whether the fluid mixture splits into two or more phases or remains in one phase using thermodynamic equilibrium approaches $[16,20,15]$. Afterwards, essential physical properties, including densities and total phase amount after splitting, can be calculated thoroughly. Especially, capillary effect, which is often ignored in conventional reservoir phase equilibrium calculation, need to be considered in shale and tight formations, as the nano-sized pores yield a large capillary pressure and confinement effect $[3,4$, $10,13,14]$. It is one of flow mechanisms for the immiscible multiphase flow especially in the formations with strong wettability preference. Surface tension is the 
main cause of capillary, and it is also greatly affected by the phase behaviors and properties of the subsurface fluids. In all, phase equilibrium calculation is the premise of accurate modeling and simulation of multiphase flow in subsurface porous media.

A number of studies have investigated the phase equilibrium problems in subsurface reservoirs, which often depend on different sets of given conditions [9, 12]. One conventional phase equilibrium calculation approach is performed under constant chemical compositions, pressure and temperature, which is known as "NPT" flash. The long history of NPT flash has brought a wide range of applications related with phase splitting calculation and stability test, but at the same time some limitations have been found regarding this method. In some specified conditions, the system equilibrium cannot be determined uniquely, or the solution from the flash calculation fails to identify a clear and determined state. For the cubic equation of state, two separate solutions need to be conducted to determine the phase molar volumes of each phase. Furthermore, the root selection procedure has to be considered in the presence of multiple roots, although the middle root is often ignored because which corresponds to a physically meaningless solution. The remaining roots need to be paired to minimize the Gibbs free energy, and the root pairing could be challengeable as there might be two roots for each phase. If the selection of pairing is wrong, the whole procedure will fail with an unstable or metastable solution. Another limitation is that pressure is not always a priori, which makes it inefficient to iteratively solve phase equilibria problems of a differernt variable specification by the NPT flash. Another approach with priori conditions including constant chemical compositions, molar volume and temperature, namely the NVT flash, has been proposed to handle the above challenges faced by NPT flash. As an alternative, it has shown great potential in compositional multiphase flow simulation in subsurface porous media.

In NVT flash calculation, solving the constrained minimization problem of total Helmholtz free energy takes the main computation consumption, and this is the main focus of designing efficient algorithms. Successive Substitution Iteration (SSI) method was first used to solve NVT flash problems by introducing a approproately defined thermodynamic function under a similar solution framework to the conventional PT-flash calculation [16]. Another popular algorithm is using Newton's method to solve the minimization problem in combination of the revised Cholesky factorization of the Hessian matrix [11,12]. Recently, an energy-stable evolution scheme is proposed for NVT flash calculations using diffuse interface models based on realistic equation of state[1,14]. In the pore scale model, partial miscibility and compressibility is considered for the first time, and the rigorous mathematical model is established based on fundamental thermodynamic laws, as well as the realistic EOS (e.g. Peng-Robinson EOS [19]). Compared to molecular simulation, which is also capable to capture the diffusive interface behaviors, this extended NVT flash scheme can save a huge portion of CPU time. 
In this paper, we will review the recent progress in the phase equilibrium calculation, which is the key step in the numerical study of compositional multiphase fluid flow in subsurface reservoirs. Compared to well-developed NPT flash, a major difference in NVT flash is to minimize Helmholtz free energy instead of Gibbs free energy. In addition, capillarity, which has been extensively taken into account for the phase equilibria problems in unconventional reservoirs, is also treated well in the algorithms. A novel convex splitting scheme of Helmholtz free energy density is proposed, to ensure the energy stability property consistent with the second law of thermodynamics. Regarding capillarity, the work done by the capillary force and the evolution equations for moles and volume are formulated carefully under rigorous mathematical derivations, with the generalized Onsager coefficient matrix.

The remainder of this paper is organized as follows. In Section 2, a general diffuse interphase model is constructed combining the NVT flash framework and the first and second laws of thermodynamics to model the multi-component multiphase fluid flow. In Section 3, an energy stable semi-implicit consistent model is presented to simultaneously solve for the phase equilibrium problem with capillarity. Numerical examples are presented in Section 4 to show the robustness and efficiency of the concluded scheme. At the end, we make some conclusions in Section 5.

\section{Diffuse Interface Model}

\subsection{Brief introduction on Helmholtz free energy}

Compared with the NPT flash, NVT flash problems introduce the Helmholtz free energy instead of the Gibbs free energy to characterize the investigated fluid systems. For a fluid mixture of $M$ components occupies volume $V$ at temperature $T$, if it is not in a stable condition, the mixture will split into two phases. We denote by $f(\mathbf{n})$ the Helmholtz free energy density which has the following form

$$
f(\mathbf{n})=f^{\text {ideal }}(\mathbf{n})+f^{\text {repulsion }}(\mathbf{n})+f^{\text {attraction }}(\mathbf{n})
$$

with

$$
\begin{aligned}
f^{\text {ideal }}(\mathbf{n}) & =R T \sum_{i=1}^{M} n_{i}\left(\ln n_{i}-1\right) \\
f^{\text {repulsion }}(\mathbf{n}) & =-n R T \ln (1-b n) \\
f^{\text {attraction }}(\mathbf{n}) & =\frac{a(T) n}{2 \sqrt{2} b} \ln \left(\frac{1+(1-\sqrt{2}) b n}{1+(1+\sqrt{2}) b n}\right)
\end{aligned}
$$

where $n=\sum_{i} n_{i}$ is the overall molar density. Consequently, in two phase flow systems, the Helmholtz free energy $F(\mathbf{n})$ can be formulated as

$$
F=f\left(\mathbf{n}_{1}\right) V_{1}+f\left(\mathbf{n}_{2}\right) V_{2}
$$


where $\mathbf{n}_{\alpha}=\mathbf{N}_{\alpha} / V_{\alpha}, \alpha=1,2$. Let $\mathbf{n}_{\alpha}=\left[n_{1, \alpha}, \ldots, n_{M, \alpha}\right]^{T}, \mathbf{N}_{\alpha}=\left[N_{1, \alpha}, \ldots, N_{M, \alpha}\right]^{T}$ and $V_{\alpha}$ represent the molar density, mole number and volume of phase $\alpha$, respectively. There are two other constraints which should be considered for NVT flash problems, namely the volume constraint $V_{1}+V_{2}=V$ and mole constraint $\mathbf{N}_{1}+\mathbf{N}_{2}=\mathbf{N}$.

\subsection{Diffuse Interface Modeling to calculate phase equilibrium}

In this subsection, a general diffuse interface model for multi-component multiphase flow is derived rigorously using the first law of thermodynamics for entropy equation and the second law of thermodynamics for the dynamic model. It can be stated from the first law of thermodynamics that:

$$
\frac{d(U+E)}{d t}=\frac{d W}{d t}+\frac{d Q}{d t}
$$

where $U$ is the internal energy, $E$ is the kinetic energy, $Q$ is the heat transfer into the system and $W$ is the work done by the force. The total entropy $S$ can be split into two contributions, one is the entropy of the system, $S_{\mathrm{sys}}$ and the entropy of the surroundings, $S_{\text {surr }}$. The relation of entropy and $Q$ can be represented by

$$
d S_{\mathrm{surr}}=-\frac{d Q}{T} .
$$

Considering the Gibbs relation $U=F+T S_{\text {sys }}$, the entropy equation could be written as:

$$
\begin{aligned}
\frac{d S}{d t} & =\frac{d S_{\mathrm{sys}}}{d t}+\frac{d S_{\mathrm{surr}}}{d t}=\frac{d S_{\mathrm{sys}}}{d t}-\frac{1}{T} \frac{d Q}{d t} \\
& =\frac{d S_{\mathrm{sys}}}{d t}-\frac{1}{T}\left(\frac{d(U+E)}{d t}-\frac{d W}{d t}\right)=-\frac{1}{T} \frac{d(F+E)}{d t}+\frac{1}{T} \frac{d W}{d t}
\end{aligned}
$$

Applying the Reynolds transport theorem and the Gauss divergence theorem, we deduce that

$$
\begin{aligned}
\frac{d S}{d t} & =\int_{V(t)} \frac{\partial s}{\partial t} d V+\int_{V(t)} \nabla(\mathbf{u} s) d V \\
\frac{d F}{d t} & =\int_{V(t)} \frac{\partial f}{\partial t} d V+\int_{V(t)} \nabla(\mathbf{u} f) d V .
\end{aligned}
$$

A symmetric matrix should exist to meet the Onsager's reciprocal principle, which is defined as $\mathbf{\Psi}=\left(\psi_{i, j}\right)_{i, j=1}^{M+1}$ and the time derivative of mole and volume will become:

$$
\frac{\partial N_{i}^{G}}{\partial t}=\sum_{j=1}^{M} \psi_{i, j}\left(\mu_{j}\left(\mathbf{n}^{L}\right)-\mu_{j}\left(\mathbf{n}^{G}\right)\right)+\psi_{i, M+1}\left(p_{G}-p_{L}-p_{c}\right), \quad 1 \leq i \leq M .
$$




$$
\frac{\partial V^{G}}{\partial t}=\sum_{j=1}^{M} \psi_{M+1, j}\left(\mu_{j}\left(\mathbf{n}^{L}\right)-\mu_{j}\left(\mathbf{n}^{G}\right)\right)+\psi_{M+1, M+1}\left(p_{G}-p_{L}-p_{c}\right) .
$$

This Onsager coefficient matrix, $\boldsymbol{\Psi}$ should be positive definite to keep the growth of total entropy, which is required by the second law of thermodynamics. One simple way to construct the matrix is to design a diagonal positive definite matrix, with the factors determined by

$$
\psi_{i, i}=\frac{D_{i} N_{i}^{t}}{R T}, i=1, \ldots, M, \quad \psi_{M+1, M+1}=\frac{C_{V}^{G} C_{V}^{L} V^{t}}{C_{V}^{L} p_{G}+C_{V}^{G} p_{L}},
$$

and the corresponding evolutionary equation for moles of component $i$ and the volume can be derived as

$$
\begin{gathered}
\frac{\partial N_{i}^{G}}{\partial t}=\frac{D_{i} N_{i}^{t}}{R T}\left(\mu_{i}\left(\mathbf{n}^{L}\right)-\mu_{i}\left(\mathbf{n}^{G}\right)\right), \quad i=1, \ldots, M, \\
\frac{\partial V^{G}}{\partial t}=\frac{C_{V}^{G} C_{V}^{L} V^{t}}{C_{V}^{L} p_{G}+C_{V}^{G} p_{L}}\left(p_{G}-p_{L}-p_{c}\right) .
\end{gathered}
$$

Here, $p_{c}$ denotes the capillary pressure, which will be explained in details in the next session. The thermodynamic stability property of this scheme is achieved on the basis of the convex-concave splitting technique [14] is used to decompose the Helmholtz free energy density as follows

$$
\begin{gathered}
f(\mathbf{n})=f^{\text {convex }}(\mathbf{n})+f^{\text {concave }}(\mathbf{n}), \\
f^{\text {convex }}(\mathbf{n})=(1+\lambda) f^{\text {ideal }}(\mathbf{n})+f^{\text {repulsion }}(\mathbf{n}), \\
f^{\text {concave }}(\mathbf{n})=f^{\text {attraction }}(\mathbf{n})-\lambda f^{\text {ideal }}(\mathbf{n}),
\end{gathered}
$$

and correspondingly the chemical potential can be expressed by computing the derivative of the Helmholtz free energy density with respect to the molar density.

\section{Phase Equilibrium Calculation considering Capillarity}

To meet the requirement in unconventional reservoir simulation, capillarity should be considered in the phase equilibrium calculations. The effects of capillary pressure on the flash calculation and reservoir simulation have been investigated thoroughly in previous literatures, with a history of more than 20 years. In 1992 [3], the vapor/liquid equilibrium (VLE) was simulated in the incorporation of the capillary pressure and a general cubic equation of state is applied. Afterwards, Peng-Robinson EOS and Soave-Redlich-Kwong EOS were used instead and different algorithms were proposed accordingly [18,21]. A wide range of temperature and pressure had been investigated, but the molecule-surface interactions were neglected, which claimed that the investigated pore size should be more than $10 \mathrm{~nm}$. However, the confinement effect ignored previously have been discovered to impact the fluid properties greatly. With the increasing 
interaction between pore walls and fluid molecules, the phase behavior in the confined space should be characterized by introducing the critical pressure and temperature shifts, which are supposed to play important roles in the accurate predictions of phase composition and behaviors in such small nanopores. The inaccurate simulation of unconventional reservoirs, either for the estimation of original oil in place or ultimate recovery prediction, has been reported to result from the failure to incorporate the capillary effect.

\subsection{Work of the capillary force}

From Session 2.2, it can be referred that the deviated phase behaviors are the result of the work done by the capillary force during the phase equilibrium process. As a result, it is crucial to quantitively model this work for the accurate prediction of the phase compositions and behaviors. The capillary pressure is defined by the difference between the pressure of wetting and nonwetting phase, $p_{c}=p_{n}-p_{w}$. If the work done by capillary force is positive, the interface between the two phases will move toward the nonwetting phase, making its volume compressed; otherwise the interface moves toward the wetting phase, which will cause the swelling of nonwetting phase. The work done by the capillary force within certain unit time is given by

$$
\frac{\mathrm{d} W}{\mathrm{~d} t}=-p_{n} \frac{\mathrm{d} V_{n}}{\mathrm{~d} t}-p_{w} \frac{\mathrm{d} V_{w}}{\mathrm{~d} t}=-p_{n} \frac{\mathrm{d} V_{n}}{\mathrm{~d} t}+p_{w} \frac{\mathrm{d} V_{n}}{\mathrm{~d} t}=-p_{c} \frac{\mathrm{d} V_{n}}{\mathrm{~d} t},
$$

assuming the capillary pressure to be constant along the interface. There are different equations to get the capillary pressure, among which commonly used one in the standalone flash calculation is the Young-Laplace equation

$$
p_{c}=\frac{2 \sigma \cos \theta}{r}
$$

and the Weinaug-Katz correlation [23]

$$
\sigma=\left[\sum_{i=1}^{M}[\mathbf{P}]_{i}\left(\mathbf{n}_{i, w}-\mathbf{n}_{i, n}\right)\right]^{4}
$$

is applied to calculate the interfacial tension $\sigma$ used in (16).

\subsection{Semi-implicit scheme}

The convex-concave splitting of Helmholtz free energy density is a popular approach to ensure the unconditional stability of numerical schemes incorporating thermodynamic dynamics in discrete formulation, as well as the same splitting approach in chemical potential. According to (12), the chemical potential $\mu_{i}(\mathbf{n})$ is supposed to have two components $\mu_{i}^{\text {convex }}(\mathbf{n})$ and $\mu_{i}^{\text {concave }}(\mathbf{n})$ as 
well, and the counterpart splitting of the chemical potential can be written as

$$
\begin{gathered}
\mu_{i}^{\text {convex }}(\mathbf{n})=\frac{\partial f^{\text {convex }}}{\partial n_{i}}=(1+\lambda) \frac{\partial f^{\text {ideal }}}{\partial n_{i}}+\frac{\partial f^{\text {repulsion }}}{\partial n_{i}} \\
\mu_{i}^{\text {concave }}(\mathbf{n})=\frac{\partial f^{\text {concave }}}{\partial n_{i}}=\frac{\partial f^{\text {attraction }}}{\partial n_{i}}-\lambda \frac{\partial f^{\text {ideal }}}{\partial n_{i}}
\end{gathered}
$$

To ensure the unconditional stability, the marching algorithm should also be treated carefully. A semi-implicit scheme, which could conserve the thermodynamic stability at the same time of easy implementation, is introduced in the algorithm. Namely, the implicit scheme is applied on the convex part of chemical potential and Helmholtz free energy density, and the explicit scheme is applied on the concave parts. In order to solve the proposed evolution equations simultaneously, a generalized Onsager coefficient matrix is introduced in the scheme. The two evolution equations, respectively for mole and volume, are solved as follows

$$
\begin{aligned}
& \frac{N_{i, 1}^{k+1}-N_{i, 1}^{k}}{\delta t}=\sum_{j=1}^{M} \psi_{i, j}\left(\mu_{j, 2}^{k+1}-\mu_{j, 1}^{k+1}\right)+\psi_{i, M+1}\left(p_{1}^{k+1}-p_{2}^{k+1}-p_{c}^{k+1}\right) \\
& \frac{V_{1}^{k+1}-V_{1}^{k}}{\delta t}=\sum_{j=1}^{M} \psi_{M+1, j}\left(\mu_{j, 2}^{k+1}-\mu_{j, 1}^{k+1}\right)+\psi_{M+1, M+1}\left(p_{1}^{k+1}-p_{2}^{k+1}-p_{c}^{k+1}\right)
\end{aligned}
$$

In the aforementioned evolution equations, the chemical potential and pressure at the $k+1$ time step are updated through

$$
\begin{aligned}
\mu_{j, 1}^{k+1} & =\mu_{j}^{\text {convex }}\left(\mathbf{n}_{1}^{k+1}\right)+\mu_{j}^{\text {concave }}\left(\mathbf{n}_{1}^{k}\right) \\
\mu_{j, 2}^{k+1} & =\mu_{j}^{\text {convex }}\left(\mathbf{n}_{2}^{k+1}\right)+\mu_{j}^{\text {concave }}\left(\mathbf{n}_{2}^{k}\right) \\
p_{1}^{k+1} & =\sum_{i=1}^{M}\left(n_{i, 1}^{k+1} \mu_{i}^{\text {convex }}\left(\mathbf{n}_{1}^{k+1}\right)+n_{i, 1}^{k} \mu_{i}^{\text {concave }}\left(\mathbf{n}_{1}^{k}\right)\right)-f^{\text {convex }}\left(\mathbf{n}_{1}^{k+1}\right)-f^{\text {concave }}\left(\mathbf{n}_{1}^{k}\right) \\
p_{2}^{k+1} & =\sum_{i=1}^{M}\left(n_{i, 2}^{k+1} \mu_{i}^{\text {convex }}\left(\mathbf{n}_{2}^{k+1}\right)+n_{i, 2}^{k} \mu_{i}^{\text {concave }}\left(\mathbf{n}_{2}^{k}\right)\right)-f^{\text {convex }}\left(\mathbf{n}_{2}^{k+1}\right)-f^{\text {concave }}\left(\mathbf{n}_{2}^{k}\right) \\
p_{c}^{k+1} & =p_{c}\left(\mathbf{N}_{1}^{k+1}, V_{1}^{k+1}\right)
\end{aligned}
$$

The unconditional stability of the above semi-implicit scheme has been proved in details in [14]. Regarding the Onsager coefficient matrix $\boldsymbol{\Psi}$, it can be divided into 4 submatrices, shown as below

$$
\boldsymbol{\Psi}=\left[\begin{array}{cc}
A & B \\
B^{T} & C
\end{array}\right]
$$


Here, $A=\partial\left(\mu_{i, 2}-\mu_{i, 1}\right) / \partial N_{i, 1}, B=\partial\left(\mu_{i, 2}-\mu_{i, 1}\right) / \partial V_{1}=\partial\left(p_{1}-p_{2}\right) / \partial N_{i, 1}$, and $C=\partial\left(p_{1}-p_{2}\right) / \partial V_{1}$. It is essential to ensure the positive definition of the Onsager coefficient matrix; otherwise, a revised Cholesky factorization will be introduced to preserve its positive definiteness. Generally, $\boldsymbol{\Psi}+\boldsymbol{E}$ should be sufficiently positive and $\boldsymbol{E}$ is added as a diagonal matrix with suitable positive entries. This positive definite property can keep the continuous increasing of entropy in the iterations, which will ensure to reach the local maximum using the Newton-Raphson method.

\section{Numerical Experiments}

The recent developed algorithms mentioned above are validated by two numerical examples. In our numerical experiments, the contact angle is set as $\theta=0$, and the investigated pore radius are $20 \mathrm{~nm}$ and $10 \mathrm{~nm}$. We assume the fluid mixture fully occupies the unit volume $V=1 \mathrm{~m}^{3}$. Parameters needed in the Peng-Robinson EOS calculation are listed in Table 1.

Table 1. Compositional properties for all studied components in numerical examples.

\begin{tabular}{lccccc}
\hline Component & $T_{\mathrm{c}, i}(\mathrm{~K})$ & $P_{\mathrm{c}, i}(\mathrm{MPa})$ & $M_{\mathrm{w}, i}(\mathrm{~g} / \mathrm{mol})$ & $\omega_{i}$ & {$[\mathbf{P}]_{i}$} \\
\hline $\mathrm{N}_{2}$ & 126.21 & 3.390 & 28.01 & 0.0390 & 41.00 \\
$\mathrm{CO}_{2}$ & 304.14 & 7.375 & 44.01 & 0.2390 & 78.00 \\
$\mathrm{C}_{1}$ & 190.56 & 4.599 & 16.04 & 0.0110 & 77.30 \\
$\mathrm{C}_{6}$ & 507.40 & 3.012 & 86.20 & 0.2960 & 271.00 \\
$\mathrm{nC}_{10}$ & 617.70 & 2.110 & 142.28 & 0.4890 & 433.50 \\
$\mathrm{PC}_{1}$ & 333.91 & 5.329 & 34.64 & 0.1113 & 97.85 \\
$\mathrm{PC}_{2}$ & 456.25 & 3.445 & 69.52 & 0.2344 & 202.52 \\
$\mathrm{PC}_{3}$ & 590.76 & 2.376 & 124.57 & 0.4470 & 356.82 \\
$\mathrm{C}_{12+}$ & 742.58 & 1.341 & 248.30 & 0.9125 & 654.97 \\
\hline
\end{tabular}

\subsection{Example 1}

In this example, we investigate the two-phase equilibrium of a ternary mixture consisting of methane $\left(\mathrm{C}_{1}\right)$, hexane $\left(\mathrm{C}_{6}\right)$ and n-decane $\left(\mathrm{nC}_{10}\right)$ with mole fractions $z_{\mathrm{C}_{1}}=0.405946, z_{\mathrm{C}_{6}}=0.297027$ and $z_{\mathrm{nC}_{10}}=0.297027$. The range of the overall molar density and the temperature under investigation is $[0,10000] \mathrm{mol} / \mathrm{m}^{3}$ and $[250,650]$ K. Simulation results of the phase equilibrium calculation are presented in Fig. 1 and Fig. 2. From Fig.1, a single two-phase region can be found in the global minimum of the TPD function at the given conditions. The solid black curve represents the boundary between the single-phase region and two-phase region. In the right figure in Fig. 1, the boundary between the vapour liquid region and single liquid region contracts at large overall concentration, which could be explained by Fig. 2. It is observed that the bulk phase envelope is reshaped by introducing the capillary pressure, which suppressed the bubble point 
curve remarkably. Meanwhile, a slight outward expansion can be found in the dewpoint curve, which is caused by the insignificant phase boundary expansion at smaller overall concentrations.
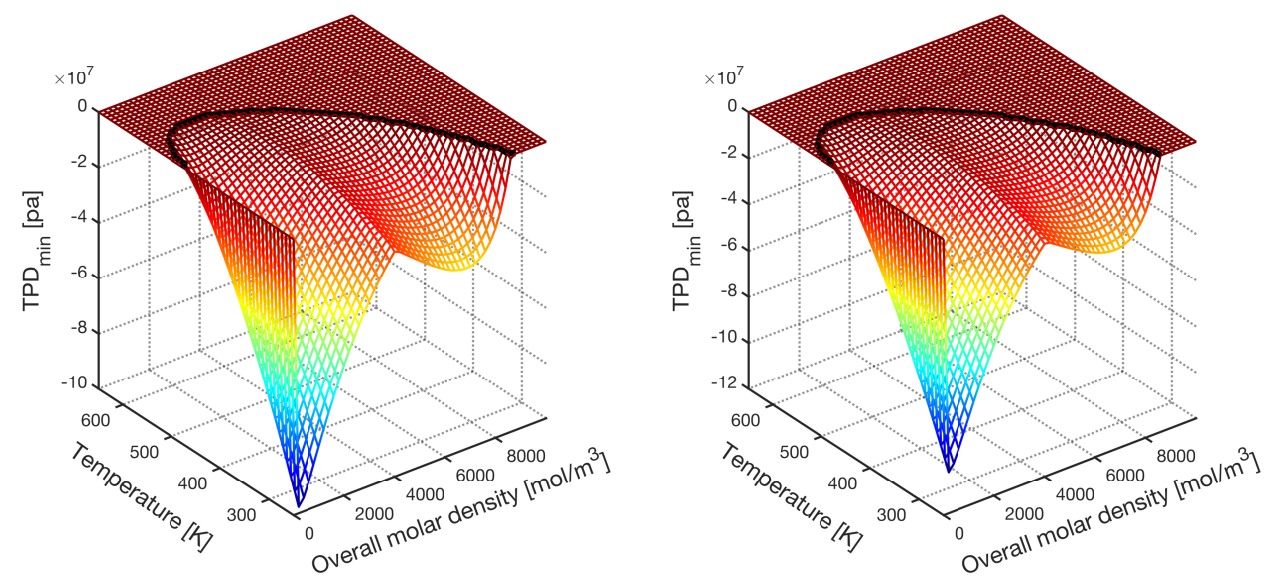

Fig. 1. Global minimum of TPD function as a function of the overall molar density and temperature for the ternary mixture of $\mathrm{C}_{1}, \mathrm{C}_{6}$ and $\mathrm{nC}_{10}$ without (left) and with (right) capillary effect, respectively.

Similar trend can be found in $[18,21]$, which can be concluded as: In the lower branch of dewpoint curve, the dew point pressure decreases in the presence of capillary pressure, while the pressure increases in the upper branch in the presence of capillary pressure. Overall, the vapor-liquid region expands due to different dew point pressure changes. Compared to the bulk phase envelope, the phase envelope at smaller pore size has more deviation. Also, the suppression of the bubble point curve is much more significant than the expansion of the dew point curve. The black point in Fig. 2 represents the approximate critical point, at which the capillary pressure is supposed to vanish due to the similar properties of both phases. It can be seen the bubble point suppression becomes larger with the thermodynamic conditions of the hydrocarbon mixture deviating away from the critical point.

\section{$4.2 \quad$ Example 2}

The second example considers a seven-component mixture consisting of nitrogen $\left(\mathrm{N}_{2}\right)$, carbon dioxide $\left(\mathrm{CO}_{2}\right)$, methane $\left(\mathrm{C}_{1}\right)$ and four hydrocarbon pseudocomponents $\mathrm{PC}_{1}, \mathrm{PC}_{2}, \mathrm{PC}_{3}$ and $\mathrm{C}_{12+}$, with mole fractions $z_{\mathrm{N}_{2}}=0.466905$, $z_{\mathrm{CO}_{2}}=0.007466, z_{\mathrm{C}_{1}}=0.300435, z_{\mathrm{PC}_{1}}=0.105051, z_{\mathrm{PC}_{2}}=0.041061, z_{\mathrm{PC}_{3}}=$ 0.045060 and $z_{\mathrm{C}_{12+}}=0.034021$. Phase equilibrium calculation for this complex fluid mixture is performed at the overall molar density $c \in[0,20000] \mathrm{mol} / \mathrm{m}^{3}$ 


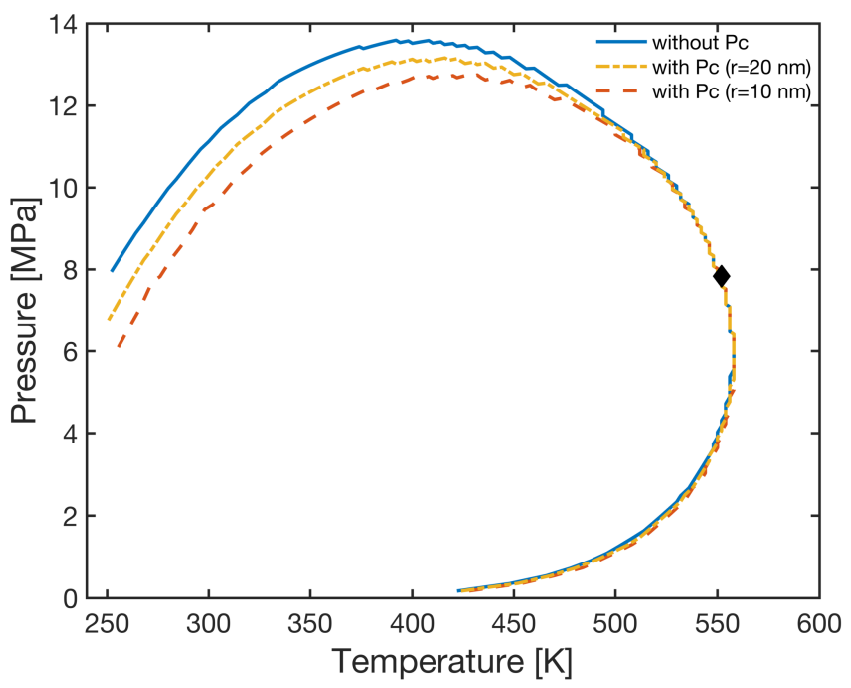

Fig. 2. Phase envelope for the ternary mixture of $\mathrm{C}_{1}, \mathrm{C}_{6}$ and $\mathrm{nC}_{10}$ at different pore radius. The black point (diamond symbol) represents the approximate critical point.

and temperature $T \in[250,650] \mathrm{K}$. Compared with the last example in [15] with different overall molar composition, this example exhibits a unique two-phase region as shown in Fig. 3, implying the equilibrium states also rely on the fluid composition. Figure 4 displays the phase envelopes of all investigated cases. No remarkable deviation from the bulk phase envelope is observed over the entire pressure and temperature windows under the specified pore radii, except a slight change at the tip of the phase envelope where the dew point curve is slightly expanded. This might be attributed to the tiny density differences between two phases under the given overall composition. As the temperature decreases, the bubble point pressure significantly increases.

\section{Conclusions}

In this paper, recent progresses on phase equilibrium calculations of subsurface fluids have been reviewed and analyzed with our own understandings. Two main different types of phase composition determination algorithms, NPT flash and NVT flash, are compared in the stability and efficiency viewpoints. Due to the limitations of the NPT flash, an alternative flash formulation at constant moles, volume and temperature is discussed, which can be used to handle the phase equilibria problems in which the pressure is not known a priori. In the proposed NVT flash calculation, a dynamic model is constructed based on diffuse interface models incorporating realistic EOS, e.g. Peng-Robinson EOS. In order to construct an unconditionally stable numerical algorithm, the total Helmholtz free energy is split by the convex-concave splitting technique, and 

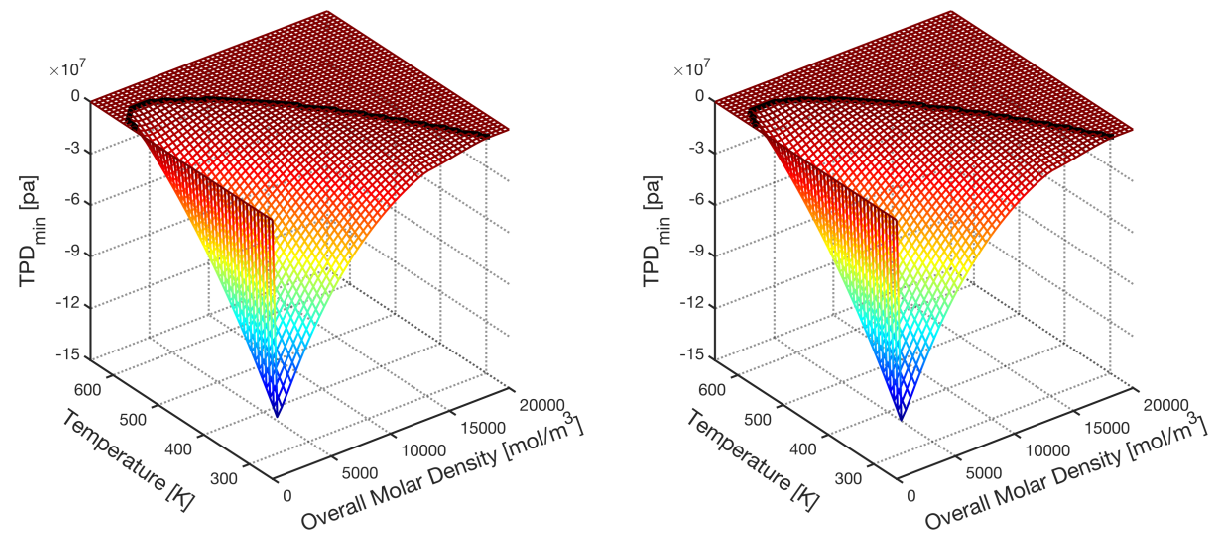

Fig. 3. Global minimum of TPD function for the seven-component mixture of $\mathrm{N}_{2}$, $\mathrm{CO}_{2}, \mathrm{C}_{1}, \mathrm{PC}_{1}, \mathrm{PC}_{2}, \mathrm{PC}_{3}$ and $\mathrm{C}_{12}+$ without (left) and with (right) capillary pressure, respectively.

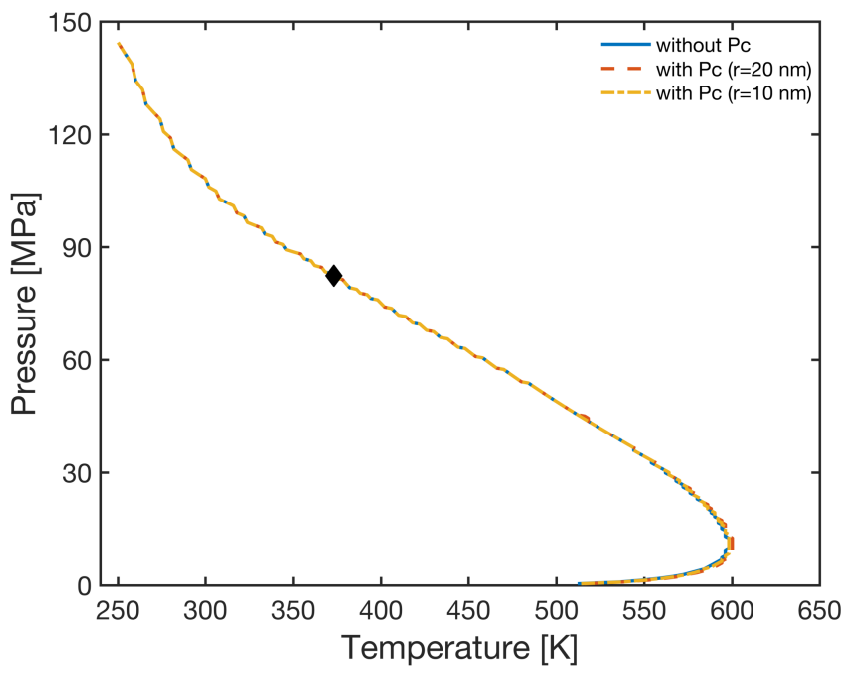

Fig. 4. Phase envelope for the seven-component mixture of $\mathrm{N}_{2}, \mathrm{CO}_{2}, \mathrm{C}_{1}, \mathrm{PC}_{1}, \mathrm{PC}_{2}$, $\mathrm{PC}_{3}$ and $\mathrm{C}_{12+}$ at different pore radius. The black point (diamond symbol) represents the approximate critical point. 
a semi-implicit scheme is introduced to simulate the dynamic model. For the simulation of phase equilibria in unconventional reservoirs, capillary pressure is taken into considerations, so as to construct a confined phase equilibrium numerical scheme. The evolution equation of mole and volume are presented to show the dynamic process to the equilibrium state from any initial condition. The proposed algorithm is tested by two numerical examples The deviated phase behavior in the presence of capillary pressure can be explained reasonably. The phase envelope will be reshaped by capillary effect and the critical point has no change. The bubble point pressure is suppressed in the presence of the capillary pressure, leading to the contraction of the phase boundary between the vaporliquid region and single fluid region. On the other hand, the phase boundary will expand towards the single vapor region caused by the dew point curve expansion. To extend our study, the confinement and complex pore size distributions will be considered in our further work to simulate more realistic phase equilibrium behaviors in porous media. Furthermore, adsorption is another important factor to determine the phase equilibria in unconventional reservoirs. Thus, the dynamic sorption should be taken into account in the optimization of the diffuse interface based models.

\section{Acknowledgements}

The authors thank for the support from the National Natural Science Foundation of China (No. 51874262) and the Research Funding from King Abdullah University of Science and Technology (KAUST) through the grants BAS/1/135101-01.

\section{References}

1. Abels, H., Garcke, H., Grün, G.: Thermodynamically consistent, frame indifferent diffuse interface models for incompressible two-phase flows with different densities. Math. Models Methods in Appl. Sci. 22(03), 1150013(1-40) (2012).

2. Aziz, K.: Petroleum reservoir simulation. Applied Science, London (1979).

3. Brusilovsky, A.I.: Mathematical simulation of phase behavior of natural multicomponent systems at high pressures with an equation of state. SPE Reserv. Eng. 7(01), 117-122 (1992).

4. Chen, Z., Huan, G., Ma, Y.: Computational methods for multiphase flows in porous media. SIAM, Philadelphia (2006).

5. Dawson, C., Sun, S., Wheeler, M.F.: Compatible algorithms for coupled flow and transport. Comput. Methods Appl. Mech. Eng. 193(23-26), 2565-2580 (2004).

6. De Groot, S.R., Mazur, P.: Non-equilibrium thermodynamics. Courier Corporation, Chelmsford (2013).

7. El-Amin, M., Sun, S., Salama, A.: Modeling and simulation of nanoparticle transport in multiphase flows in porous media: CO2 sequestration. Math. Methods Fluid Dyn. Simul. Giant Oil Gas Reserv. (2012).

8. Espinoza, D.N., Santamarina, J.C.: Water-CO2-mineral systems: Interfacial tension, contact angle, and diffusion-Implications to CO2 geological storage. Water Resour. Res. 46(7), W07537(1-10) (2010). 
9. Firoozabadi, A.: Thermodynamics of hydrocarbon reservoirs. McGraw-Hill, New York (1999).

10. Ho, C.K., Arnold, B.W., Altman, S.J.: Dual-Permeability Modeling of Capillary Diversion and Drift Shadow Effects in Unsaturated Fractured Rock. J. Heat Transfer 131(10), 101012(1-6) (2009).

11. Jindrová, T., Mikyška, J.: Fast and robust algorithm for calculation of two-phase equilibria at given volume, temperature, and moles. Fluid Phase Equilib. 353, 101114 (2013).

12. Jindrová, T., Mikyška, J.: General algorithm for multiphase equilibria calculation at given volume, temperature, and moles. Fluid Phase Equilib. 393, 7-25 (2015).

13. Kou, J., Sun, S.: A new treatment of capillarity to improve the stability of IMPES two-phase flow formulation. Comput. \& Fluids 39(10), 1923-1931 (2010).

14. Kou, J. Sun, S.: A stable algorithm for calculating phase equilibria with capillarity at specified moles, volume and temperature using a dynamic model. Fluid Phase Equilib. 456, 7-24 (2018).

15. Li, Y., Kou, J., Sun, S.: Thermodynamically Stable Two-Phase Equilibrium Calculation of Hydrocarbon Mixtures with Capillary Pressure. Ind. Eng. Chem. Res. 57(50), 17276-17288 (2018).

16. Mikyška, J., Firoozabadi, A.: A new thermodynamic function for phasesplitting at constant temperature, moles, and volume. AIChE J. 57(7), 1897-1904 (2011).

17. Moortgat, J., Sun, S., Firoozabadi, A.: Compositional modeling of threephase flow with gravity using higherorder finite element methods. Water Resour. Res. 47(5) W05511(1-26) (2011).

18. Nojabaei, B., Johns, R.T., Chu, L.: Effect of capillary pressure on phase behavior in tight rocks and shales. SPE Reserv. Eval. Eng. 16, 281-289 (2013).

19. Peng, D.Y., Robinson, D.B.: A new two-constant equation of state. Ind. Eng. Chem. Fundam. 15, 59-64 (1976).

20. Polívka, O., Mikyška, J.: Compositional modeling in porous media using constant volume flash and flux computation without the need for phase identification. J. Comput. Phys. 272, 149-169 (2014).

21. Sandoval, D.R., Yan, W., Michelsen, M.L., Stenby, E.H.: The phase envelope of multicomponent mixtures in the presence of a capillary pressure difference. Ind. Eng. Chem. Res. 55, 6530-6538 (2016).

22. Sun, S., Liu, J.: A locally conservative finite element method based on piecewise constant enrichment of the continuous Galerkin method. SIAM J. Sci. Comput. 31(4), 2528-2548 (2009).

23. Weinaug, C.F., Katz, D.L.: Surface tensions of methane-propane mixtures. Ind. Eng. Chem. 35(2), 239-246 (1943).

24. Wu, Y.S., Qin, G.: A generalized numerical approach for modeling multiphase flow and transport in fractured porous media. Commun. Comput. Phys. 6(1), 85-108 (2009).

25. Tao Zhang, Jisheng Kou, Shuyu Sun. Advances in Geo-Energy Research. 2017, 1(2) : 124-134 